\title{
Multi-Electrode Samples for TEM Studies of Corrosion
}

\author{
D.S. Elswick*, J.J. Hren*, P.G. Kotula**, F.D. Wall** \\ * Department of Materials Science and Engineering, North Carolina State University, Raleigh NC \\ **Sandia National Laboratory, Albuquerque, NM
}

Localized corrosion initiation of high purity aluminum was studied using high-resolution microanalysis. Two sample geometries, high purity Al needles and multi-electrode wires embedded in epoxy, were developed to localize the initiation process. Small area electrodes were utilized to observe local changes in the oxide before and after polarization to the pitting potential. The multielectrode samples consist of a cylindrical array of small, parallel Al wires, $125 \mu \mathrm{m}$ in diameter, embedded in epoxy. One end of the wire was prepared through a mechanical polish followed by an electropolish, while the other end of the wire provided an electrical contact for experimentation. Electrochemical exposures were performed on both sample geometries, followed by detailed characterization and analyses of the oxide films, the oxide-Al interface, and the Al substrate. TEM preparation was successful for this sample geometry through the use of dual-beam FIB technology.

Analytical techniques included transmission electron microscopy (TEM) for both structural characterization and chemical microanalysis. Energy-filtered imaging and x-ray spectral imaging characterized the structure of both the oxide and the Al substrate. The initial oxide was found to be uniform with a thickness of 4-5 nm for both sample geometries, as seen in Figure 1. Note that there is no significant change in oxide thickness over the range of surface orientations present in the needle (Figure 1a) nor across the grain boundary (Figure 1b) in the flat specimen.

The multi-electrode wires were addressed individually to pitting potential experiments and then examined using TEM. Figure 2 shows the result of one wire exposed to $0.05 \mathrm{M} \mathrm{NaCl}$ aq solution at room temperature with a constant potential scan of $0.1667 \mathrm{mV} / \mathrm{s}$ up to the pitting potential. A single pit formed as seen in Figure 2a. The TEM bright field image in Figure $2 b$ shows that the oxide/hydroxide has apparently thickened by a factor of $\sim 10$ and contains voids. Other results, from similar electrochemical testing, show a thickening and change in morphology of the oxide/hydroxide due to changes in both the chemical and electrical environments.

Jump ratio imaging has proved a valuable tool for this study, yet jump ratio images of these same oxides did not convincingly demonstrate the presence of chlorine. X-ray spectral image analysis [utilizing a fine probe to map out the spectra] indicated a $\mathrm{Cl}^{-}$signal was present and that it was incorporated into the amorphous oxide/hydroxide structure. Figure 3a is a dark field STEM image of the cross-section through the pit. The box indicates the area used for spectral imaging. The results in Figure $3 \mathrm{~b}$ suggests that chlorine plays a role in the corrosion process, but that the concentration levels have not yet been spatially localized. Attempts to obtain full electron energy loss spectroscopy (EELS) scans have not succeeded to date, due primarily to specimen damage at $300 \mathrm{keV}$. Further experiments will be attempted at $100 \mathrm{keV}$ where it is hoped that radiation effects can be greatly reduced.

Sandia is a multiprogram laboratory operated by Sandia Corporation, a Lockheed Martin Company, for the U.S. Dept. of Energy under Contract DE-AC04-94AL85000. 


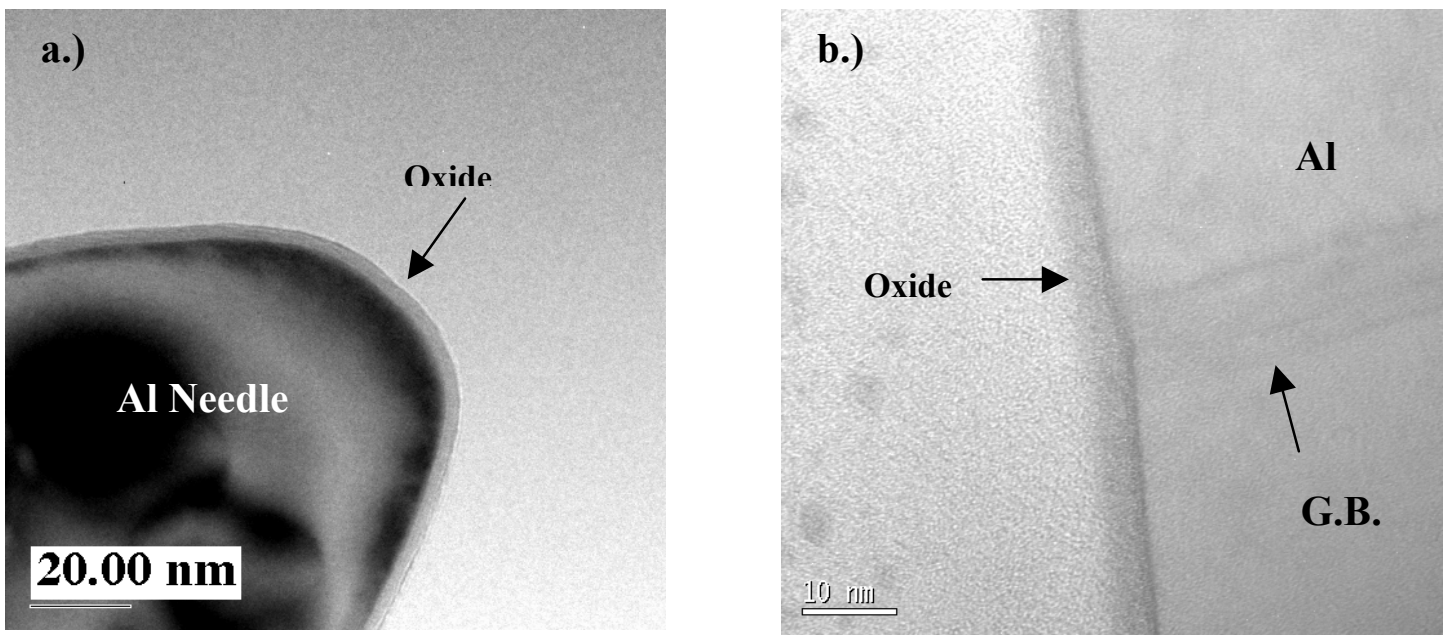

Figure 1. Uniform initial oxide; 4-5 nm thickness a.) Needle geometry b.) Flat sample.
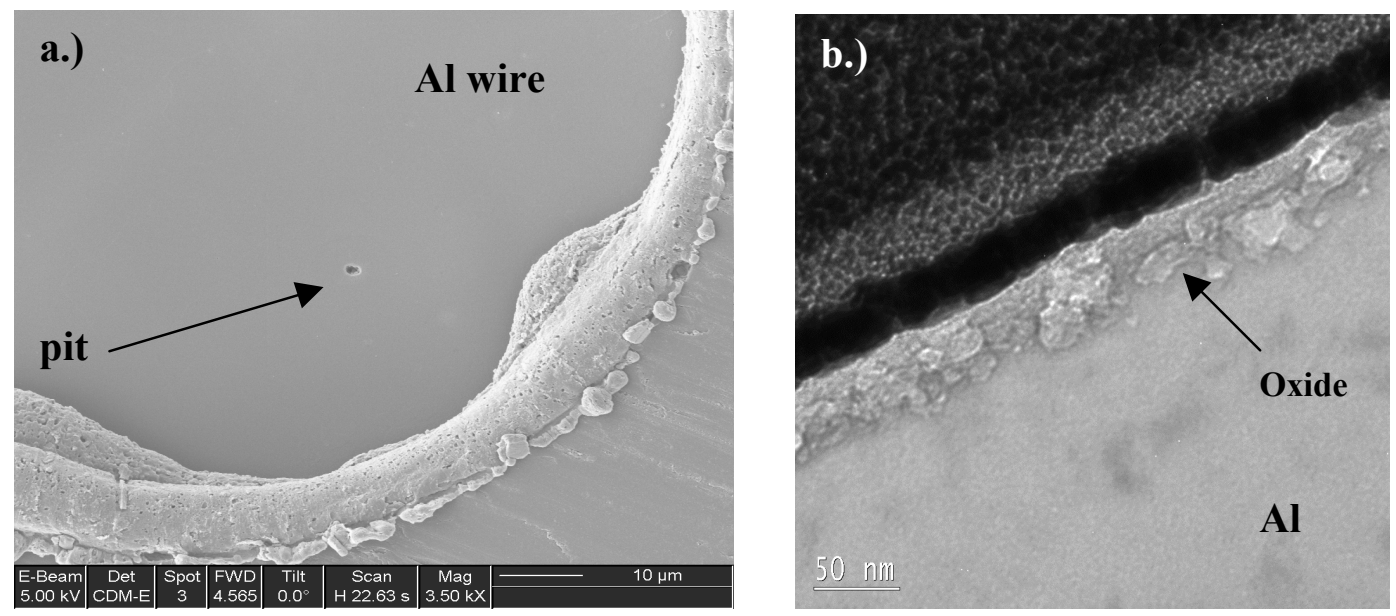

Figure 2. Multi-electrode sample exposed to pitting potential experiment. a.) SEM image of single pit formed. FIB slice for TEM was through the pit b.) TEM bright field image of surface area $1 \mu$ away from pit.
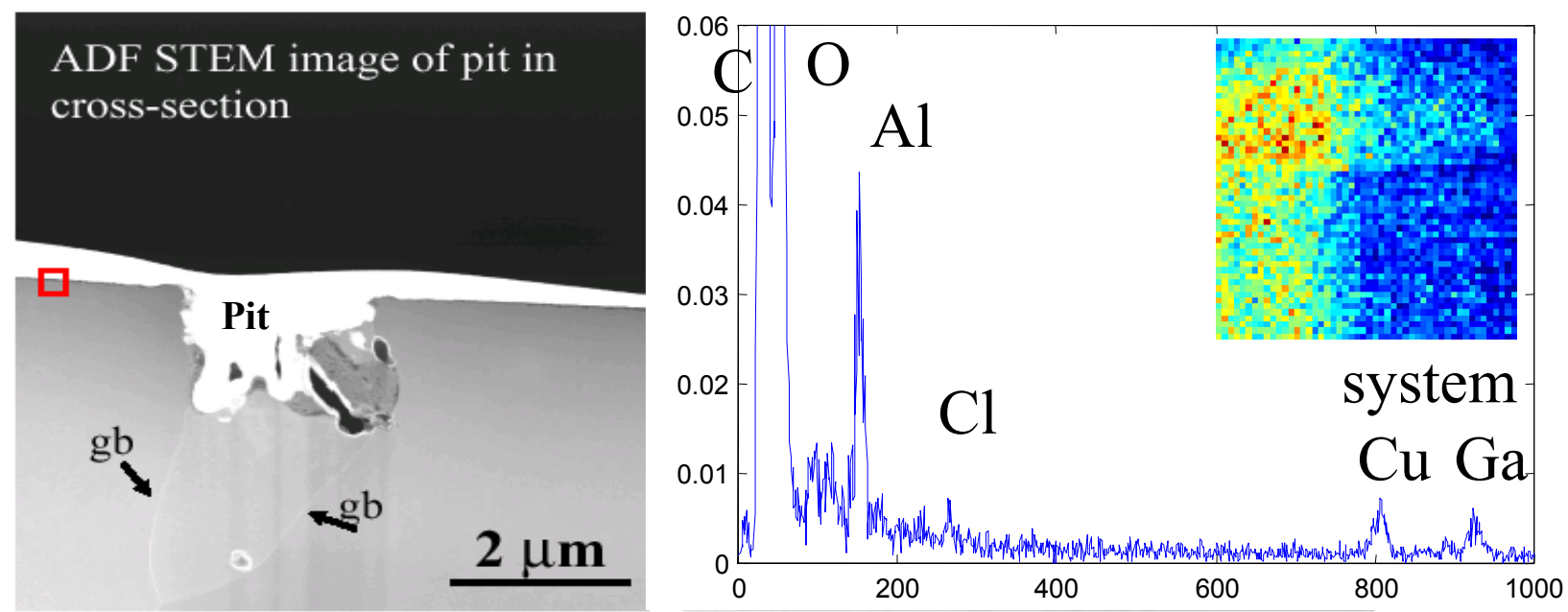

Figure 3. a.) STEM ADF image of pitted sample. The box indicates a $50 \mathrm{~nm} \times 50 \mathrm{~nm}$ region selected in STEM mode for Automated X-ray Spectrum Imaging Analysis (AXSIA). b.) AXSIA data showing a faint $\mathrm{Cl}$ signal. 